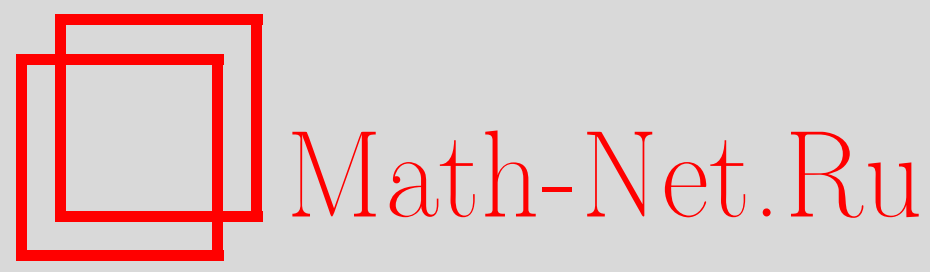

И. А. Солдатенков, О достаточных условиях сходимости последовательных приближений, Матем. заметки, 1999, том 65, выпуск 6, 854-859

DOI: https://doi.org/10.4213/mzm1121

Использование Общероссийского математического портала Math-Net.Ru подразумевает, что вы прочитали и согласны с пользовательским соглашением http://www . mathnet.ru/rus/agreement

Параметры загрузки:

IP: 52.23 .180 .231

26 апреля 2023 г., 05:33:10

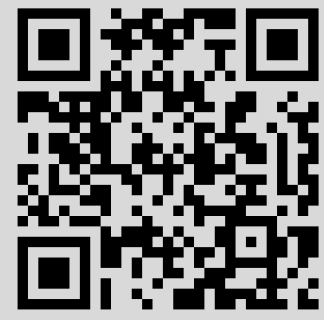




\section{О ДОСТАТОЧНЫХ УСЛОВИЯХ СХОДИМОСТИ ПОСЛЕДОВАТЕЛЬНЫХ ПРИБЛИЖЕНИЙ}

\section{И. А. Солдатенков}

Получены достаточные условия сходимости последовательных приближений к решению операторного уравнения. Продемонстрировано использование полученных условий применительно к нелинейному интегральному уравнению типа Гаммерштейна.

Библиограффия: 6 названий.

1. Введение. Излагаемый ниже материал касается условий сходимости последовательных приближений $u_{n}$ (простых итераций)

$$
u_{n+1}=\widehat{R} u_{n}, \quad n=0,1, \ldots,
$$

к решению $u \in U$ уравнения

$$
u=\widehat{R} u,
$$

где $\widehat{R}$ - оператор, отображающий пространство $U$ в себя, $u_{0} \in U$. Дадим обзор некоторых результатов по данному вопросу.

Классическим является принцип Банаха неподвижной точки [1], [2], утверждающий, что последовательность (1) сходится к единственному решению $u \in U$ уравнения (2) при любом $u_{0} \in U$, если только $U$ - полное метрическое пространство, а $\widehat{R}$ является сжимающим в $U$, т.е. для любых $u^{(1)}, u^{(2)}$ из $U$ :

$$
\rho\left(\widehat{R} u^{(1)}, \widehat{R} u^{(2)}\right) \leqslant q \rho\left(u^{(1)}, u^{(2)}\right), \quad q \in[0,1),
$$

где $\rho$ - символ метрики в $U$.

Требование сжимаемости оператора $\widehat{R}$ на всем пространстве $U$, присутствующее в принципе неподвижной точки, может быть ослаблено. В теоремах из [3], [4] вводится некоторое подмножество пространства $U$, на котором выполняется $(3)$ и формулируется правило выбора начального приближения $u_{0}$, порождающего сходящуюся к решению уравнения (2) последовательность (1).

Другое обобщение [3] классического принципа неподвижной точки допускает нелинейную зависимость определенного вида правой части неравенства $(3)$ от $\rho\left(u^{(1)}, u^{(2)}\right)$.

Описываемое ниже условие сходимости последовательных приближений также допускает нелинейность в правой части (3) и предлагает достаточно конструктивное правило выбора начального приближения.

Работа выполнена при финансовой поддержке Российского фонда фундаментальных исследований, грант № 98-01-00901. 
2. Основные утверждения. Далее будем считать, что $U$ - банахово пространство, причем $\rho\left(u^{(1)}, u^{(2)}\right)=\left\|u^{(1)}-u^{(2)}\right\|$. Имеет место

ЛЕмма А. Пусть, исходя из некоторого $u_{0}$, построена бесконечная последовательность $\left\{u_{n}\right\}$ вида (1) такая, что для любых ее әлементов $u_{n}, u_{n+1}$ выполняется неравенство

$$
\left\|\widehat{R} u_{n+1}-\widehat{R} u_{n}\right\| \leqslant q\left(\left\|u_{n}\right\|,\left\|u_{n+1}-u_{n}\right\|\right)\left\|u_{n+1}-u_{n}\right\|
$$

где $q(X, Y)$ - некоторая функиия. Тогда последовательность $\left\{u_{n}\right\}$ является фундаментальной, если

a) $q(X, Y)$ возрастает nо $X \geqslant 0 u Y \geqslant 0$;

б) найдутся $\lambda \in[0,1) u h \geqslant\left\|u_{1}-u_{0}\right\|$ maкuе, что

$$
q\left(\left\|u_{0}\right\|+\frac{h}{1-\lambda}, h\right)=\lambda
$$

ДокАЗАТЕЛЬСТВо. Положим $\delta_{n}=\left\|u_{n+1}-u_{n}\right\|$. Используя метод математической индукции, установим, что для любого натурального $i$ справедливо неравенство

$$
\delta_{i} \leqslant \lambda \delta_{i-1}, \quad i=1,2, \ldots,
$$

при этом ввиду $\delta_{0} \leqslant h, \lambda \in[0,1)$ будут вьполняться и неравенства

$$
\delta_{i} \leqslant \lambda^{i} \delta_{0} \leqslant \lambda^{i} h \leqslant h, \quad i=1,2, \ldots
$$

При $i=1$, основьваясь на определении (1) последовательности $\left\{u_{n}\right\}$, неравенстве (4) и условиях а), б) леммы А, можно записать

$$
\delta_{1}=\left\|\widehat{R} u_{1}-\widehat{R} u_{0}\right\| \leqslant q\left(\left\|u_{0}\right\|, \delta_{0}\right) \delta_{0} \leqslant q\left(\left\|u_{0}\right\|+\frac{h}{1-\lambda}, h\right) \delta_{0}=\lambda \delta_{0}
$$

т.е. имеем (6) для $i=1$.

Предположим теперь, что неравенство (6) и, следовательно, (7) справедливы для $i=$ $1, \ldots, n$. Покажем, что тогда (6) вьполняется при $i=n+1$.

Запишем аналогично (8):

$$
\delta_{n+1}=\left\|\widehat{R} u_{n+1}-\widehat{R} u_{n}\right\| \leqslant q\left(\left\|u_{n}\right\|, \delta_{n}\right) \delta_{n}
$$

причем в силу справедливости неравенств (6) и (7) при $i=1, \ldots, n$ имеют место соотношения

$$
\begin{aligned}
\left\|u_{n}\right\| & =\left\|u_{n}-u_{n-1}+u_{n-1}-\cdots-u_{0}+u_{0}\right\| \leqslant\left\|u_{0}\right\|+\delta_{0}+\cdots+\delta_{n-1} \\
& \leqslant\left\|u_{0}\right\|+\lambda^{0} h+\cdots+\lambda^{n-1} h \leqslant\left\|u_{0}\right\|+\frac{h}{1-\lambda} .
\end{aligned}
$$


Учитывая эти соотношения, а также неравенство (7) при $i=n$ и условия а), б), неравенство (9) можно продолжить

$$
\delta_{n+1} \leqslant q\left(\left\|u_{0}\right\|+\frac{h}{1-\lambda}, h\right) \delta_{n}=\lambda \delta_{n}
$$

тем самым установив справедливость (6) при $i=n+1$.

Таким образом, неравенства (6) и, следовательно, (7) имеют место при любом $i \geqslant 1$. Основьваясь на данном результате, возьмем произвольные натуральные $N, n>N$, $m>n$ и запишем по аналогии с [1], [2] неравенства

$$
\begin{aligned}
\left\|u_{m}-u_{n}\right\| & =\left\|u_{m}-u_{m-1}+u_{m-1}-\cdots+u_{n+1}-u_{n}\right\| \leqslant \delta_{m-1}+\cdots+\delta_{n} \\
& \leqslant \lambda^{m-1} h+\cdots+\lambda^{n} h \leqslant \frac{\lambda^{n} h}{1-\lambda} \leqslant \frac{\lambda^{N} h}{1-\lambda},
\end{aligned}
$$

правая часть которых стремится к 0 при $N \rightarrow \infty$, а это по определению означает, что $\left\{u_{n}\right\}$ - фундаментальная последовательность. Лемма доказана.

Лемма А позволяет доказать основную теорему.

Теорема А. Пусть оператор $\widehat{R}$ отображсает банахово пространство $U$ в себя и для любых әлементов $u^{(1)}, u^{(2)}$ из $U$ выполнено

$$
\left\|\widehat{R} u^{(2)}-\widehat{R} u^{(1)}\right\| \leqslant q\left(\left\|u^{(1)}\right\|,\left\|u^{(2)}-u^{(1)}\right\|\right)\left\|u^{(2)}-u^{(1)}\right\| .
$$

Тогда если при некотором $u_{0} \in U$ условия а) $и$ б) леммы А выполняются, то в U существует решение уравнения (2), к которому сходятся последовательные приблихсения (1) при выбранном $u_{0}$.

ДокАЗАТЕЛЬСТво. При условиях теоремы для $u_{0} \in U$ может быть построена последовательность $\left\{u_{n}\right\}$ вида (1), удовлетворяющая условиям леммы А и, поэтому, являющаяся фундаментальной. Последнее по определению полного пространства означает существование предела последовательности $\left\{u_{n}\right\}$ :

$$
u=\lim _{n \rightarrow \infty} u_{n} \in U
$$

который является решением уравнения (2). Действительно, в силу (10) вьполняется неравенство

$$
\left\|u_{n+1}-\widehat{R} u\right\|=\left\|\widehat{R} u_{n}-\widehat{R} u\right\| \leqslant q\left(\|u\|,\left\|u_{n}-u\right\|\right)\left\|u_{n}-u\right\|
$$

правая часть которого стремится к 0 при $n \rightarrow \infty$, поскольку имеет место (11) и функция $q(X, Y)$ убьвает при $Y \rightarrow 0$. Таким образом, с учетом $(11)$ можно записать

$$
\widehat{R} u=\lim _{n \rightarrow \infty} u_{n+1}=u \text {. }
$$

Теорема доказана. 
ЗАмЕчаниЕ 1 . В частном случае $q(X, Y)=$ const равенство (5) дает $q=\lambda \in[0,1)$, в результате чего условие (10) теоремы А совпадает с определением (3) сжимающего оператора. Последнее означает эквивалентность принципа неподвижной точки и теоремы А при $q(X, Y)=$ const.

ЗАмечаниЕ 2. Неравенство (10), в отличие от (3) при $q \in[0,1)$ [1], не обеспечивает единственности решения уравнения (2). Для того чтобы единственность имела место, достаточно наложить на функцию $q(X, Y)$ дополнительное ограничение: $q(X, Y) \in$ $[0,1)$ при $X \geqslant 0, Y \geqslant 0$.

Действительно, в этом случае если $u^{(1)}$ и $u^{(2)}$ - решения $(2)$, то по аналогии с [1], [2] можно записать неравенство

$$
\left\|u^{(2)}-u^{(1)}\right\|=\left\|\widehat{R} u^{(2)}-\widehat{R} u^{(1)}\right\| \leqslant q\left(\left\|u^{(1)}\right\|,\left\|u^{(2)}-u^{(1)}\right\|\right)\left\|u^{(2)}-u^{(1)}\right\|,
$$

которое вьполняется только при $\left\|u^{(2)}-u^{(1)}\right\|=0$.

ЗАМЕчАнИЕ 3 . Функция $q(X, Y)$ определяется неравенством $(10)$ и, очевидно, не является единственной.

3. ПРИмЕР 1. В качестве тривиального примера использования теоремы А рассмотрим числовое уравнение

$$
u=E(u) \equiv \widehat{R} u, \quad u \in(-\infty, \infty)
$$

Здесь в качестве пространства $U$ выступает множество действительных чисел с нормой $\|u\|=|u|$, которое, как известно, является полным. Очевидно, что оператор $\widehat{R}$ вида (12) отображает пространство $U$ в себя.

Пусть $E^{\prime}(t) \in C(-\infty, \infty)$, а также существует возрастающая по $t \geqslant 0$ функция $E_{+}^{\prime}(t)$ такая, что

$$
E_{+}^{\prime}(t) \geqslant \max \left\{\left|E^{\prime}(-t)\right|,\left|E^{\prime}(t)\right|\right\}
$$

При этих условиях для оператора (12) можно получить оценку типа (10) с функцией

$$
q(X, Y)=E_{+}^{\prime}(X+Y)
$$

которая в силу определения (13) является возрастающей по $X \geqslant 0$ и $Y \geqslant 0$.

Таким образом, условия теоремы А для уравнения (12) оказываются вьполненными, и это уравнение имеет решение, к которому сходятся приближения (1), если только соответствующее начальное приближение $u_{0}$ удовлетворяет равенству $(5)$, имеющему согласно (14) вид

$$
E_{+}^{\prime}\left(\left|u_{0}\right|+\frac{2-\lambda}{1-\lambda} h\right)=\lambda, \quad \lambda \in[0,1),
$$

где $h \geqslant\left|\widehat{R} u_{0}-u_{0}\right|$.

Так если $E(t)=k t^{2}+b$, то, полагая $E_{+}^{\prime}(t)=2 k|t|, u_{0}=0, h=|b|$, получим из (15) следующее уравнение:

$$
2 k|b|\left(\frac{2-\lambda}{1-\lambda}\right)=\lambda \text {, }
$$


которое имеет решение $\lambda \in[0,1)$, если

$$
2 k|b| \in[0,3-\sqrt{8}] .
$$

Соотношение (16) является достаточным условием существования решения уравнения (12) с квадратичной правой частью, к которому сходятся приближения (1) при $u_{0}=0$.

Заметим, что путем непосредственного решения квадратного уравнения $u=k u^{2}+b$ можно получить необходимое и достаточное условие существования действительных корней этого уравнения в виде неравенства $2 k b \leqslant 1 / 2$, которому, в частности, удовлетворяет соотношение (16).

ПримеР 2. Рассмотрим решение уравнения типа Гаммерштейна [5]

$$
u(x)=-\int_{a}^{b} K(x, y) E(u(y)) d y+f(x) \equiv(\widehat{R} u)(x)
$$

в банаховом пространстве $U=C[a, b]$ с нормой

$$
\|u\|=\max _{x \in[a, b]}|u(x)| .
$$

Будем считать, что $E(t) \in C^{1}(-\infty, \infty), f(x) \in C[a, b]$, тогда как ядро $K(x, y)$ является непрерывным на $\Pi=[a, b] \times[a, b]$ кроме, быть может, множества точек, образующих непрерьвную линию $y=\psi(x), x \in[a, b]$, на которой $K(x, y)$ может принимать бесконечные значения. Кроме того, будем предполагать, что

$$
\int_{a}^{b}|K(x, y)| d y \rightrightarrows \sigma(x), \quad x \in[a, b]
$$

т.е. имеет место равномерная по $x \in[a, b]$ сходимость интеграла от $|K(x, y)|$ к некоторой функции $\sigma(x)$, являющейся, как нетрудно установить, непрерьвной на $[a, b][6]$.

Несложные выкладки показьвают, что при наложенных выше ограничениях на функции $E(t), f(x)$ и $K(x, y)$ оператор вида (17) отображает пространство $U=C[a, b]$ в себя $[6]$.

В целях использования теоремы А наложим на функцию $E(t)$ дополнительное ограничение, а именно: предположим, что существует возрастающая по $t \geqslant 0$ функция $E_{+}^{\prime}(t)$, определяемая неравенством (13). Теперь нетрудно получить оценку

$$
\left|E\left(u^{(2)}(x)\right)-E\left(u^{(1)}(x)\right)\right| \leqslant E_{+}^{\prime}\left(\left\|u^{(1)}\right\|+\left\|u^{(2)}-u^{(1)}\right\|\right)\left|u^{(2)}(x)-u^{(1)}(x)\right|,
$$

которая в свою очередь позволяет записать неравенство типа (10) с функцией

$$
q(X, Y)=\sigma_{m} E_{+}^{\prime}(X+Y)
$$

где

$$
\sigma_{m}=\max _{x \in[a, b]} \sigma(x)
$$

причем в силу (13) такая функция является возрастающей по $X \geqslant 0$ и $Y \geqslant 0$. 
В результате условия теоремы А для уравнения (17) оказьваются вьполненными, и это уравнение имеет решение, к которому сходятся приближения (1), если только соответствующее начальное приближение $u_{0}(x)$ удовлетворяет равенству $(5)$, имеющему согласно (18) вид

$$
\sigma_{m} E_{+}^{\prime}\left(\left\|u_{0}\right\|+\frac{2-\lambda}{1-\lambda} h\right)=\lambda
$$

где $h \geqslant\left\|\widehat{R} u_{0}-u_{0}\right\|, \lambda \in[0,1)$.

Так если $E(t)=|t|^{\gamma}, \gamma \geqslant 1$, то, полагая $E_{+}^{\prime}(t)=\gamma|t|^{\gamma-1}, u_{0}(x) \equiv 0, h=\|f\|$, получим из (19) следующее уравнение для $\lambda \in[0,1)$ :

$$
B\left(\frac{2-\lambda}{1-\lambda}\right)^{\gamma-1}=\lambda
$$

причем $B=\sigma_{m} \gamma\|f\|^{\gamma-1}$.

Несложньй анализ показывает, что уравнение (20) имеет решение $\lambda \in[0,1)$, если

$$
B \in\left(0, B_{*}(\gamma)\right]
$$

где

$$
B_{*}(\gamma)=z\left(\frac{1-z}{2-z}\right)^{\gamma-1}, \quad z=\frac{1}{2}\left(\gamma+2-\sqrt{(\gamma+2)^{2}-8}\right)
$$

Таким образом, если вьполняется условие (21), то последовательные приближения (1) при $u_{0}(x) \equiv 0$ сходятся к решению уравнения $(17)$ со степенной функцией $E(t)$ указанного выше вида.

\section{СПИСОК ЦИТИРОВАННОЙ ЛИТЕРАТУРЫ}

[1] Натансон И. П. Теория функций вещественной переменной. М.: Наука, 1974.

[2] Садовничий В. А. Теория операторов. М.: Изд. МГУ, 1986.

[3] Коллатц Л. Функциональный анализ и вычислительная математика. М.: Мир, 1969.

[4] Березин И. С., ЖКидков Н. П. Методы вычислений. Т. 1. М.: Наука, 1966.

[5] Трикоми Ф. Интегральные уравнения. М.: ИЛ, 1960.

[6] Солдатенков И. А. О некоторых достаточных условиях сходимости последовательных приближений. Препринт ИПМ РАН № 580. М.: ИПМ РАН, 1997. 